(c) 2017, THE AUTHORS. Published by FASS and Elsevier Inc. on behalf of the American Dairy Science Association ${ }^{\circledR}$.

This is an open access article under the CC BY-NC-ND license (http://creativecommons.org/licenses/by-nc-nd/3.0/).

\title{
Comparison of a commercially available oral nutritional supplement and intravenous fluid therapy for dehydration in dairy calves
}

\author{
Jared D. Taylor, ${ }^{1}$ Merel Rodenburg, ${ }^{2}$ and Timothy A. Snider \\ Department of Veterinary Pathobiology, Center for Veterinary Health Sciences, Oklahoma State University, Stillwater 74078-2007
}

\begin{abstract}
Calf scours is a primary cause of morbidity and mortality in the dairy industry. Effective treatments are needed to minimize death, maximize welfare, and maintain growth and productivity. The objective of this trial was to compare the efficacy of a commercially available nutritional supplement (Diaque, Boehringer-Ingelheim Vetmedica Inc., St. Joseph, MO) and i.v. lactated Ringer's solution (LRS) in rehydrating, preventing acidemia, and correcting electrolyte imbalances in an experimental model for calf scours. Twenty-four colostrum-fed suckling dairy calves were used in a modified crossover design. An osmotic diarrhea was induced by orally feeding commercial milk replacer modified with high level of sucrose to create a hypertonic milk solution, and administering oral hydrochlorothiazide and spironolactone for $48 \mathrm{~h}$. The intention was to create a challenge sufficient to result in moderately dehydrated, standing calves without producing severe depression or loss of suckle. The efficacy of i.v. fluid therapy and a commercial nutritional supplement were subsequently compared for reversing the effects of the diarrheal disease. Treatment A consisted of administering the nutritional supplement according to label directions (100 g in $1.9 \mathrm{~L}$ of warm water, 3 times a day), and treatment B consisted of i.v. LRS (2 L, once a day). Clinical signs and laboratory results were obtained once daily by a blinded observer. The induction method was effective in creating the desired effect, as demonstrated by weight loss and subjective health and hydration scores. Both treatment groups experienced increases in body weight, base excess, and bicarbonate, and decreases in total protein and packed cell volume following treatment. Both i.v. LRS and Diaque are effective methods to correct hypovolemia and control derangements in acidbase status in calves with diarrhea and dehydration.
\end{abstract}

Received October 24, 2016.

Accepted February 16, 2017.

${ }^{1}$ Corresponding author: jared.d.taylor@okstate.edu

${ }^{2}$ Current address is 301 Lakeview Terrace, Clovis, NM 88101.
Key words: calf scours, diarrhea, oral rehydration therapy, fluid therapy

\section{INTRODUCTION}

Calf scours accounts for the majority of preweaned dairy calf morbidity and mortality (USDA, 2007) and is second only to respiratory disease in morbidity of beef calves (USDA, 2010). The economic effect of diarrheal disease in calves is not limited to mortality, but includes the cost of treatment as well as notable negative effects on calf growth and future performance (Donovan et al., 1998).

Diarrhea in young calves is multifactorial and affected by management, hygiene, immune status, and nutrition of the calf, as well as burden of infectious agents (Foster and Smith, 2009). The most common pathogens are enterotoxigenic Escherichia coli, Cryptosporidium parvum, rotavirus, and coronavirus. However, mixed etiology is common in field conditions, and attempts to distinguish between agents can be unrewarding. If left untreated, dehydration subsequent to scours can lead to decreased appetite, electrolyte and metabolic imbalances, and increased susceptibility to secondary disease. Although antimicrobial and other ancillary therapy may be warranted in calves showing systemic illness (Constable, 2009; Smith, 2015), the mainstay of treatment entails addressing losses of fluids and electrolytes.

Successful treatment depends upon early detection and intervention, and a protocol that not only rehydrates calves but also maintains hydration and electrolyte balance in the face of ongoing losses. An ideal treatment for dehydrated, diarrheic calves achieves this while being minimally labor and time intensive, easy to administer with little risk of user error or insult to calf, and part of a protocol that is straightforward and easily trainable to employees (McGuirk, 2008).

Intravenous fluids have been proven as an effective treatment for diarrheic calves (Constable, 2003). However, administration is time consuming, invasive, and requires a relatively high level of employee training and skill. Oral rehydration therapy (ORT) has been a long 
accepted alternative to i.v. fluid therapy. Extensive variability is present in ORT products on the market, and thus products should not be assumed to be equally effective in rehydrating and correcting electrolyte and acid-base imbalances. Current recommendations state that an ORT product should meet the following criteria (Smith, 2009): supply sufficient sodium to normalize the extracellular fluid volume; provide agents (glucose, citrate, acetate, and so on) to facilitate absorption of sodium and water from the intestine; provide an alkalinizing agent (acetate, propionate, or bicarbonate) to correct or maintain acid-base status; and provide energy, because most calves that have diarrhea are in a negative energy balance.

The commercially available product Diaque (Boehringer-Ingelheim Vetmedica Inc., St. Joseph, MO) is an oral rehydration nutritional supplement that satisfies these criteria, while also containing additional nutrients and constituents (including protein, fat, and additional glucose). If this commercially available product is comparable to i.v. fluid therapy in treatment of diarrheic, moderately dehydrated calves, use of the product instead of i.v. therapy would be beneficial to the beef and dairy calf raising industry. This could give producers a viable, less labor-intensive option that helps achieve all of the goals of successful calf diarrhea and dehydration management. The null hypothesis was that i.v. fluids would be more effective than an oral supplement in rehydrating and maintaining acid/base and electrolyte balance in diarrheic calves. The objective of this study was to compare the efficacy of a nutritional supplement ORT to i.v. administered lactated Ringer's solution (LRS) in rehydrating, maintaining acid-base balance, and limiting electrolyte derangements in moderately dehydrated, diarrheic calves.

\section{MATERIALS AND METHODS}

\section{Calves}

Twenty-four healthy, colostrum-fed 2- to 12-d-old dairy bull and freemartin calves were used. They were transported from Dalhart, Texas, to Stillwater, Oklahoma, and housed at the Oklahoma State University Laboratory Animal Research Center. Calves were housed in groups of 2 to 4 in an indoor, forced-air climate controlled environment throughout the study. Calves were fed milk replacer by bottle twice daily at a volume equal to approximately $10 \%$ of the mean BW per day. All animals had ad libitum access to starter feed throughout the project, and access to water during acclimation and wash-out periods. Calves were given at least $3 \mathrm{~d}$ of acclimation between transport and onset of the initial induction procedure. The study was conducted in conformance with university institutional animal care and use policies (animal care and use protocol VM 14-26).

\section{Experimental Design}

The number of observations was based upon intention to assess noninferiority of oral supplement compared with i.v. fluids, with the following assumptions: $\alpha$ of 0.05 , power of 0.8 , and a difference between treatments (as measured in BW change from initiation of treatment to end of study) equal to the standard deviation of effect (in this case, estimated to be $1.2 \mathrm{~kg}$, or approximately $3 \%$ of mean BW). Enrollment of 24 calves allowed for maintaining a power of 0.8 despite loss of follow-up due to death or withdrawal of 8 observational periods. The study was designed as a crossover with a 7-d washout period between study periods. Calves were initially divided into 2 groups. One group underwent induction and treatment (first trial period), whereas the other group remained unenrolled. Upon completion of the first study period, the second group underwent induction and treatment (second study period), whereas the first group completed a washout period. Calves in the first group then re-enrolled for the third study period, whereas the second group was in washout. Due to health complications (some related to study protocol and others not), not all calves were able to participate in the period in which they were initially intended to be enrolled. In those cases, the calf was eligible for enrollment in the next study period following resolution of health issues. This resulted in modifications from a true crossover design, and necessitated a fifth study period. Calves within a group were initially blocked by BW, then randomly assigned to treatment for their first trial period. Each calf was then enrolled in the opposite treatment for its subsequent period. In general, calves underwent 2 study periods, one for each treatment. However, 4 calves were used in 3 study periods. When this was the case, assignment of the calf to the treatment group for the third treatment was done randomly.

Treatment protocol A consisted of administering a nutritional supplement (Diaque, see Table 1 for nutrient analysis) reconstituted per label directions (100 g in $1.9 \mathrm{~L}$ warm water) by bottle every 8 to $10 \mathrm{~h}$ (3 times a day) for 2 consecutive days. Treatment protocol B consisted of $2 \mathrm{~L}$ of LRS administered i.v. in a bolus infusion once a day for 2 consecutive days (Figure 1). Calves receiving LRS were restrained in lateral recumbency and the skin over a jugular vein was scrubbed with chlorhexidine-saline preparation solution and sprayed with alcohol before placement of a 14- or 16ga, 1.5 inch $(3.81 \mathrm{~cm})$ needle. Skin over the jugular vein was clipped in all animals to facilitate blood collection 


$\begin{array}{ll}\text { Event 1 } & \text { Event 2 } \\ \text { - Initial physical exam } & \text { - Dehydration confirmed } \\ \text { and data collection } & \text { - May occur 24 or 48 h } \\ \text { - Physical exam and } & \text { after event 1 } \\ \text { data confirm health } & \text { - Physical exam and } \\ \text { status and provide } & \text { data collection } \\ \text { baseline } & \text { - If calf not dehydrated at } \\ \text { - Dehydration } & \text { 24 h, protocol continues } \\ \text { protocol begins after } & \text { - If calf not dehydrated at } \\ \text { event 1 } & \text { 48 h, calf is removed } \\ & \text { - Diuresis terminates but } \\ & \text { sucrose continues } \\ & \text { - Treatment begins 12 } \\ & \text { h after event 2 }\end{array}$

\section{Treatment}

- LRS group receives $2 \mathrm{~L}$ of LRS IV SID, approx. 18 and $42 \mathrm{~h}$ after event 2

- Diaque group receives $1.9 \mathrm{~L}$ of reconstituted product $\mathrm{PO}$ TID, approx. 10, 16, 22, 34, 40 , and $46 \mathrm{~h}$ after event 2

\section{Event 3}

- Occurs 48 h after event 2

- Treatment is complete

- Sucrose is terminated

- Physical exam and data collection

- No results reported
Event 4

- Occurs 24 h after event 3

- Final physical exam and data collection

- No sucrose or treatment has been received for $>24 \mathrm{~h}$

- Calves deemed as returning to health

- Trial termination

Figure 1. Major activities and timelines for each event. LRS = lactated Ringer's solution; SID = once a day; TID = 3 times a day; PO = orally.

and fluid administration while maintaining blinding of study personnel.

Any calf that failed to consume $50 \%$ or more of a given nutritional supplement treatment, or $50 \%$ or more of the day's milk replacer (combined refusal from both feedings $>50 \%$ ) was administered the remaining volume via esophageal feeder.

\section{Induction Protocol}

An osmotic diarrhea was induced over a 24 - to 48 -h period according to a protocol described by Walker et al. (1998) with modifications. A hypertonic solution of milk replacer and sucrose was fed to all calves enrolled in the induction phase. Commercial milk replacer (Nutra blend 22-20, Nutra Blend LLC, Neosho, MO; the same as used throughout the study) was prepared according to directions, then mixed 1:1 with a $20 \%$ sucrose solution. The sucrose-milk replacer mixture was fed at a volume of approximately $5 \%$ BW per feeding (twice a day), delivering approximately $5 \mathrm{~g} / \mathrm{kg}$ of

Table 1. Guaranteed analysis of Diaque ${ }^{1}$ nutritional supplement per 100-g serving (calves in the study received 3 servings per day, per label)

\begin{tabular}{lcc}
\hline Component & $\begin{array}{c}\text { Minimum, if } \\
\text { applicable } \\
(\%)\end{array}$ & $\begin{array}{c}\text { Maximum, if } \\
\text { applicable } \\
(\%)\end{array}$ \\
\hline CP & 2.2 & \\
Crude fat & 4.6 & 4.5 \\
Crude fiber & 0.06 & 5.3 \\
Phosphorus & 4.5 & 5.0 \\
Salt & 3.4 & 15.8 \\
Sodium & 1.0 & \\
Potassium & & \\
Ash & 53.5 &
\end{tabular}

${ }^{1}$ Diaque (Boehringer-Ingelheim Vetmedica Inc., St. Joseph, MO). sucrose per feeding, in addition to half-strength milk replacer. Frequency of feeding the hypertonic mixture was done as needed to induce and maintain acceptable fecal scores. Fecal character was subjectively assessed by an investigator (either JT or TS) before each feeding, and if fecal scores of the group as a whole were deemed adequately loose (watery, with little to no firm material in a pen), standard milk replacer solution was fed at approximately $5 \%$ of BW per feeding. If many stools visible in the pen had a more normal consistency, the $20 \%$ sucrose solution was added 1:1 to milk replacer. Administration of sucrose was continued, as needed, throughout the treatment period and fecal consistency was allowed to return to normal only after the final treatment had been administered. Hydrochlorothiazide (1 mg/kg, orally) and spironolactone ( $2 \mathrm{mg} / \mathrm{kg}$, orally) were administered every $8 \mathrm{~h}$ during induction, until the calf was classified as adequately dehydrated and enrolled in treatment. Once a calf was enrolled, administration of diuretics was ceased. The hydration status of calves was monitored each evening. Calves were enrolled either 24 or $48 \mathrm{~h}$ after initiation of the dehydration protocol, based upon the cumulative assessment of BW loss (targeting approximately 7\%), moderate skin tenting, recession of eye globe within the orbit, and mild to moderate depression. Assessment was done by a single investigator (MR). Calves that were not adequately dehydrated $48 \mathrm{~h}$ after initiation of the trial period were excluded from that period.

\section{Data Collection}

Pretreatment, treatment, and posttreatment data collection was done for each calf. Parameters measured at each assessment included BW, skin tenting, recession of eye globe within the socket, mentation, fecal score, total serum protein level (measured via optical 
refractometer following centrifugation), and packed cell volume (PCV). In addition, sodium, potassium, and bicarbonate levels and base excess were measured via handheld instrument (i-Stat, Abbott Point of Care Inc., Princeton, NJ) immediately before initiation of induction, at time of enrollment to treatment (either 24 or $48 \mathrm{~h}$ postinduction), each evening during treatment, and through one day after completion of treatment. The handheld instrument and disposable cartridges are commercially available, and directly measure sodium and potassium levels, while calculating base excess and bicarbonate values. All sample and data collections throughout the trial were performed by an individual blinded to treatment (MR). A total of 40 complete observations (from initiation through $1 \mathrm{~d}$ posttreatment) were collected and used for statistical analysis. This was equal to the number desired based on the original power calculation.

\section{Statistical Analysis}

Mentation score, eye socket recession, and skin tent were combined to form a composite dehydration score. Efficacy of dehydration protocol was assessed by comparing analytes within a calf over time. This was done either via Wilcoxon rank sums (for nonnormally distributed measures) or paired $t$-test (for normally distributed measures). Kruskal Wallis one-way ANOVA was used to compare the composite dehydration scores and base excess values from initial enrollment to beginning of treatment and after completion of treatment, whereas ANOVA for repeated measures was used for analyzing TP, PCV, and electrolytes within a calf over time. When treatments were compared, the Wilcoxon ranked sum or 2 -sample $t$-test with equal variances assumed was used.

\section{RESULTS}

No significant differences were present between groups for BW $(P=0.68)$, dehydration assessment scores $(P=$ 0.87 ), or serum analytes (all $P>0.7$ except for $\mathrm{HCO}_{3}$, which was $P=0.44$ ) before initiation of the dehydration protocol. The administration of sucrose and the 2 diuretic agents resulted in profuse, watery diarrhea, with subsequent moderate dehydration. Per protocol, this was achieved in almost all cases without evidence of profound depression, recumbency, or loss of a suckle reflex. Calves experienced an average BW loss of approximately $7.9 \%$ following the induction protocol (range of $3 \%$ gain to $13.9 \%$ loss). Mean BW before induction (i.e., at initial assessment) was $40.0 \mathrm{~kg}$ (range 27.6-61.3) versus $36.7 \mathrm{~kg}$ (range 27.5-53) at treatment enrollment (after induction but before initiation of treatment).
This decrease in BW was statistically significant $(P<$ 0.0005 ). Serum total protein and PCV values were increased at enrollment compared with initial assessment, also consistent with dehydration. Mean total protein at initial assessment was $5.64 \mathrm{~g} / \mathrm{dL}$ (range 4.6-7.2) compared with 6.18 after dehydration, at time of enrollment for treatment (range 5-7.4; $P<0.0005$ ). Mean PCV was $35 \%$ (range 20-48) and increased to $38.4 \%$ (range 23-48; $P<0.0005)$ at enrollment. Mean calculated base excess at initial assessment was $7.5 \mathrm{mEq} / \mathrm{L}$ (range -5 to 21 ); and at enrollment decreased to 5.7 [range -5 to 12 ; $P=0.017$ for all calves; no significant difference from initial to enrollment for the LRS group $(P=0.288)]$. Mean bicarbonate concentration at initial assessment was $32.06 \mathrm{mEq} / \mathrm{L}$ (range 21-44.4) and at treatment enrollment decreased to 30.38 [range 21.7-36.3; $P=$ 0.001 for all calves; no significant difference between initial assessment and time of enrollment for the LRS group $(P=0.18)]$. In contrast, $\mathrm{Na}^{+}$and $\mathrm{K}^{+}$values were not significantly different after dehydration but before treatment, compared with initial evaluation (138 vs. $137 \mathrm{mEq} / \mathrm{L}, P=0.43$; and 5.0 vs. $4.9 \mathrm{mEq} / \mathrm{L}, P=$ 0.134). A significant increase was observed in $\mathrm{BW}$ from time of treatment enrollment to completion of the trial (1 d after completion of treatment) for both treatment groups. Mean weight of the supplement group at treatment enrollment was $36.9 \mathrm{~kg}$ (range 27.9-53.1) compared with a mean weight at trial completion of 39.2 (range 27.2-60.6; $P<0.0005$ ). The mean weight of the LRS group at enrollment was $36.6 \mathrm{~kg}$ (range 27.5-52.8) and at completion was $38.8 \mathrm{~kg}$ (range 28.5-60.6; $P$ $<0.0005)$. No difference was observed between the 2 treatment groups in final $\mathrm{BW}$ or change in $\mathrm{BW}$ from induction to completion $(P=0.55)$. Similarly, total protein values, PCV, base excess, and bicarbonate all showed significant changes toward baseline from treatment enrollment to completion of the trial test period (see Table 2). No significant difference was observed in these values between the 2 treatment groups. No significant difference was observed in $\mathrm{Na}^{+}$or $\mathrm{K}^{+}$, either between induction and final, or between the 2 treatment protocols. Major findings are summarized in Table 2.

\section{DISCUSSION}

Based on the study reported here, both i.v. LRS and a commercially available nutritional supplement are effective treatments for rehydrating and mitigating acid-base changes in moderately dehydrated, diarrheic calves. Both treatment groups experienced increases in BW, normalizations in base excess and bicarbonate, and decreases in total protein and PCV during and upon completion of treatment. These improvements occurred in the face of continued challenge with osmotic 
diarrhea throughout the treatment period. Induction of diarrhea was discontinued at the time of treatment completion, to simulate typical management of treating calves until resolution of clinical disease.

Comparisons between induction and initial evaluation parameters confirm that the induction protocol was successful in replicating at least some of the aspects of natural disease, including diarrhea, dehydration, and a shift in acid-base status. The most extreme and notable response to the protocol was observed in BW. Calves demonstrated an average of $7.9 \%$ decrease in BW from the time of initiation of the induction protocol to enrollment for treatment. However, an extremely wide range of BW change was observed (from a $3 \%$ increase to a $14 \%$ decrease). Many factors can contribute to relatively rapid changes in $\mathrm{BW}$, including alterations in gut fill and prior hydration status. Change in gut fill almost assuredly influenced the most extreme observations, as a $13 \%$ loss of BW solely or primarily attributable to fluid loss would result in severe dehydration and likely death. Similarly, the weight gain observed in a very small number of calves could simply reflect an accumulation of fluid in the bowels due to the hyperosmolar environment and before onset of severe diarrhea. This is in no small part a byproduct of the chosen induction protocol and would not be expected in natural disease. Recognizing these limitations, BW was not the only parameter measured to assess hydration status. Subjective dehydration scores increased from a median of 1 before induction of dehydration to a median of 3 before treatment. This was on a scale of 0 to 9 , but included mentation, which by design, should have never increased above 1. Other parameters, including PCV, total protein, bicarbonate, and base excess, were also significantly different from baseline after the induction protocol and before enrollment for treatment, all in a manner consistent with dehydration. Moreover, these same parameters were significantly different following treatment compared with before initiating treatment, confirming success of both treatment approaches in addressing the dehydration and metabolic derangements. Treatment effectively returned bicarbonate, base excess, and PCV to values not significantly different from the initial baseline. Body weight remained significantly lower after treatment than baseline $(P=0.001)$ when all calves were examined; however, the LRS group was not significantly different from baseline $1 \mathrm{~d}$ after completion of the trial $(P=0.07)$. Body weight $1 \mathrm{~d}$ after completion of the trial was significantly higher than after induction protocol and before treatment began $(P$ $<0.0005)$. Total protein was also lower than baseline following treatment $(P<0.0005)$. This may reflect loss of serum protein through the diarrheal process, consumption/decay of passively acquired antibodies, or may simply reflect dehydration that was present before the induction process that was corrected with treatment. This last consideration cannot be overlooked, as previous and concurrent natural challenge by various infectious agents could have contributed to some degree of initial dehydration, particularly for early study

Table 2. Values of various parameters for the 2 treatment groups, at initiation of dehydration (event 1), initiation of treatment (event 2), and $1 \mathrm{~d}$ after completion of treatment (event 4)

\begin{tabular}{|c|c|c|c|}
\hline Parameter $^{1}$ & Event 1 & Event 2 & Event 4 \\
\hline $\begin{array}{l}\text { BW Diaque mean }(\mathrm{kg}) \\
\text { (Range) }\end{array}$ & $\begin{array}{l}40.4^{\mathrm{a}} \\
(29.0 \text { to } 61.3)\end{array}$ & $\begin{array}{l}36.9^{\mathrm{b}} \\
(27.9 \text { to } 53.1)\end{array}$ & $\begin{array}{l}39.5^{\mathrm{c}} \\
(28.5 \text { to } 60.6)\end{array}$ \\
\hline BW LRS mean (kg) & $39.5^{\mathrm{a}}$ & $36.6^{\mathrm{b}}$ & $38.8^{\mathrm{a}}$ \\
\hline (Range) & $(27.6$ to 57.2$)$ & $(27.5$ to 52.8$)$ & $(27.2$ to 56.3$)$ \\
\hline $\begin{array}{l}\text { Total protein Diaque mean (g/dL) } \\
\text { (Range) }\end{array}$ & $\begin{array}{l}5.7^{\mathrm{a}} \\
(4.6 \text { to } 7.2)\end{array}$ & $\begin{array}{l}6.2^{\mathrm{b}} \\
(5.0 \text { to } 7.2)\end{array}$ & $\begin{array}{l}5.4^{\mathrm{c}} \\
(4.3 \text { to } 6.7)\end{array}$ \\
\hline $\begin{array}{l}\text { Total protein LRS mean }(\mathrm{g} / \mathrm{dL}) \\
\text { (Range) }\end{array}$ & $\begin{array}{l}5.6^{\mathrm{a}} \\
(4.8 \text { to } 6.6)\end{array}$ & $\begin{array}{l}6.1^{\mathrm{b}} \\
(5.0 \text { to } 7.4)\end{array}$ & $\begin{array}{l}5.4^{\mathrm{c}} \\
(4.3 \text { to } 6.7)\end{array}$ \\
\hline $\begin{array}{l}\text { Packed cell volume Diaque mean (\%) } \\
\text { (Range) }\end{array}$ & $\begin{array}{l}34.5^{\mathrm{a}} \\
(20 \text { to } 48)\end{array}$ & $\begin{array}{l}38.9^{\mathrm{b}} \\
(25 \text { to } 48)\end{array}$ & $\begin{array}{l}35.1^{\mathrm{c}} \\
(24 \text { to } 43)\end{array}$ \\
\hline $\begin{array}{l}\text { Packed cell volume LRS mean (\%) } \\
\text { (Range) }\end{array}$ & $\begin{array}{l}34.8^{\mathrm{a}} \\
(21 \text { to } 45)\end{array}$ & $\begin{array}{l}37.8^{\mathrm{b}} \\
(23 \text { to } 47)\end{array}$ & $\begin{array}{l}34.4^{\mathrm{c}} \\
(21 \text { to } 45)\end{array}$ \\
\hline $\begin{array}{l}\text { Base excess Diaque mean } \\
\text { (Range) }\end{array}$ & $\begin{array}{l}7.6^{\mathrm{a}} \\
(1 \text { to } 21)\end{array}$ & $\begin{aligned} & 5.1^{\mathrm{b}} \\
&(-5 \text { to } 12)\end{aligned}$ & $\begin{array}{l}8.5^{\mathrm{a}} \\
(2 \text { to } 16)\end{array}$ \\
\hline $\begin{array}{l}\text { Base excess LRS mean } \\
\text { (Range) }\end{array}$ & $\begin{array}{l}7.4^{\mathrm{ab}} \\
(-5 \text { to } 13)\end{array}$ & $\begin{array}{c}6.3^{\mathrm{a}} \\
(-2 \text { to } 11)\end{array}$ & $\begin{array}{l}9.2^{\mathrm{b}} \\
(3 \text { to } 13)\end{array}$ \\
\hline $\begin{array}{l}\mathrm{HCO}_{3} \text { Diaque mean }(\mathrm{mEq} / \mathrm{L}) \\
\text { (Range) }\end{array}$ & $\begin{array}{l}32.1^{\mathrm{a}} \\
(25.2 \text { to } 44.2)\end{array}$ & $\begin{array}{l}30.0^{\mathrm{b}} \\
(21.7 \text { to } 36.3)\end{array}$ & $\begin{array}{l}32.3^{\mathrm{a}} \\
(27.4 \text { to } 38.7)\end{array}$ \\
\hline $\begin{array}{l}\mathrm{HCO}_{3} \mathrm{LRS} \text { mean }(\mathrm{mEq} / \mathrm{L}) \\
\text { (Range) }\end{array}$ & $\begin{array}{l}32.1^{\mathrm{ab}} \\
(25.2 \text { to } 44.2)\end{array}$ & $\begin{array}{l}30.0^{\mathrm{a}} \\
(21.7 \text { to } 36.3)\end{array}$ & $\begin{array}{l}32.3^{\mathrm{b}} \\
(27.4 \text { to } 38.7)\end{array}$ \\
\hline
\end{tabular}

${ }^{\mathrm{a}-\mathrm{c}}$ Means within a row with different superscripts differ $(P<0.05)$. No significant differences existed between treatments.

${ }^{1}$ Diaque (Boehringer-Ingelheim Vetmedica Inc., St. Joseph, MO); LRS = lactated Ringer's solution. 
periods. This is supported by the fact that some calves were deemed too ill to include in their initially planned period, and were retained and treated to include in a later period when they had recovered. Some such affected calves remained in protocol, as they met the moderately dehydrated, standing, and suckling criteria. No uneven distribution of ill calves existed between the 2 treatment groups. Only 2 calf mortalities occurred while enrolled in a trial period, one due to a clostridial abomasitis presumably induced by the supra-physiologic sucrose intake, and the second resulting from severe dehydration and failure to adequately respond to treatment. Although the intention was to elicit only a moderate dehydration, the mortality caused by the induction protocol confirms the physiologic burden placed upon the calves by the induction process.

In addition to correcting dehydration, a major objective of treatment for scouring calves is maintaining appropriate acid-base status. Significant changes in serum bicarbonate values and base excess show that both treatments were successful in reversing the mild metabolic imbalance. Metabolic acidosis secondary to scours results from a combination of factors. These include fecal loss of bicarbonate, decreased tissue perfusion and hypoxia leading to anaerobic glucose metabolism and subsequent L-lactate production, decreased renal efficiency in excretion of excess hydrogen ions, as well as D-lactate and L-lactate absorption from increased bacterial fermentation of nutrients in the distal gut (Kasari, 1999). Maintenance of acid-base balance is critical in treating calves with scours due to the association of acidemia with depression and loss of suckle, as well as hypothermia, shock, and coma, if severe (Naylor, 1999). In general, calves in the study did not develop acidemia, as indicated by calculated base excess or bicarbonate values. Nonetheless, both treatments served to assist in maintaining these parameters within normal range, including facilitating statistically significant changes from initiation to conclusion of treatment. Unresolved acidemia in scouring calves is associated with depression, loss of suckle, and recumbency (Kasari and Naylor, 1986; Nakagawa et al., 2007). For an orally administered product to be effective and practical, a suckle reflex must be present; therefore, it is imperative that metabolic acidosis be mild and quickly controlled for ORT to be a treatment option. Results reported here support the appropriateness of both treatments in preventing acidemia, as all calves except for one that died retained strength and ability to stand and suckle throughout the treatment periods.

Neither the induction protocol nor either treatment protocol showed significant effects on measured electrolyte levels. This is consistent with other reports using a similar model (Leal et al., 2012). Electrolyte derange- ments in scouring calves can be variable and difficult to predict without individual analysis (Michell et al., 1992). Correction of hydration status and electrolyte loss and improving ECF volume are key components of a rehydration protocol. Although the absence of significant changes during the induction protocol makes it impossible to assess efficacy of either treatment in correcting derangements, both the nutritional supplement and i.v. LRS appeared adequate to assist maintenance of normal electrolyte parameters throughout and following the treatment period.

The experimental model used in this trial identified and treated calves that were only moderately dehydrated. These calves expressed observable clinical signs of dehydration and BW loss, but did not suffer derangements sufficient to cause severe electrolyte or metabolic imbalances, or the systemic manifestations of such imbalances. Further studies may be warranted to assess and compare the efficacy of the nutritional supplement examined here in correcting electrolyte imbalances in more severely dehydrated calves. However, ORT is generally considered a viable treatment option only for calves still able to stand and demonstrate a suckle reflex; these 2 features are often absent in more severe disease (Smith and Berchtold, 2014).

Induction of an osmotic diarrhea is not a perfect replication of natural disease. It is likely that there is less injury to the gastrointestinal mucosa with an induced disease, meaning that absorption of oral fluids may be less efficient in natural disease. However, use of calves affected by natural disease in a study such as this is fraught with difficulties. The specific etiology is typically unknown in natural disease, and the challenge burden, insult to the gut, and risk to the calf is unknowable and inconsistent. Use of calves suffering natural disease would have also precluded a crossover study design. Given these considerations, it was decided to use a well-established and proven experimental induction model. Further study is warranted to assess efficacy of the nutritional supplement in natural disease.

A 7-d washout period was chosen to permit calves' digestive tract to return to normal and for calves to achieve normal hydration status between challenge episodes. The length of the interval was arbitrary but seemed more than adequate, as stool was typically normal by 25 to $36 \mathrm{~h}$ after the final sucrose feeding. The subjective scoring at time of enrollment suggested that calves were adequately rehydrated before entering the next trial period.

The volume of fluids provided by the 2 treatments were notably different $(5.7 \mathrm{~L}$ daily for the nutritional supplement, and $2 \mathrm{~L}$ for the i.v. treatment). This may lead to questions regarding the appropriateness of comparing the 2 approaches. The study design at- 
tempted to account for several considerations which make the difference in volumes appropriate. First, it was important to follow manufacturer's recommendations for the nutritional supplement. Second, we sought to be consistent with practices in the field for either therapy. Intravenous fluid therapy is primarily used in the field for calves more severely affected than those in our study. However, large calf ranches often prefer to use i.v. fluids as a first-line treatment under the assumption it is more effective than orally administered products. Even in these situations, i.v. treatment is typically only feasible once a day. The study protocol reflected this limitation. Similarly, administration of more than $2 \mathrm{~L}$ in a short period of time would pose a risk of fluid overload, particularly in hypoproteinemic calves. Volume overload via oral administration is much less likely to occur, and is almost inconceivable with a balanced electrolyte product such as used in this report. Moreover, absorption of fluids from the gut will be less than $100 \%$, particularly in the face of diarrheal disease. Thus, a larger volume is justified for oral treatment, as compared with i.v.

Lactated Ringer's solution was used as the i.v. fluid of choice, as it is a commercially available and commonly used replacement fluid. It is a poly-ionic fluid that more closely mirrors physiologic fluids than does $0.9 \%$ sodium chloride. The inclusion of lactate provides a substrate that serves as an alkalizing agent following metabolism in the liver. This is beneficial in reducing the acidosis typical in scouring calves, whereas $0.9 \%$ sodium chloride provides a strong cation-anion ratio that exerts an acidifying effect. Although other commercially available products provide slightly higher sodium content along with metabolizable bases, the difference is generally deemed clinically insignificant with no distinction between products such as LRS, Normosol R (Pfizer, New York, NY), or Plasma-Lyte (Baxter, Deerfield, IL). Although there is limited evidence comparing the efficacy of these balanced solutions, currently little clinical difference is seen between them (Noritomi et al., 2011). Severely acidotic calves benefit from isotonic or hypertonic sodium bicarbonate, but acetated or lactated Ringer's solution is preferred for mildly affected calves (Berchtold, 2009).

The crossover study design allowed calves to be used in multiple study periods throughout the trial. This meant that a wide age gap (up to $25 \mathrm{~d}$ ) was present between the first and the last periods. Diarrhea-associated dehydration is of concern to all preweaned calves, and the study was designed to encompass all susceptible ages of calves. However, the small number of calves in each period precludes analysis by age group. Further research is warranted to study variation in efficacy of treatment in different calf age groups.
Labor and facilities limitations necessitated a less than optimal schedule for feeding and supplement delivery. Calves received milk replacer between 0830 and $0930 \mathrm{~h}$, and again between 1600 and $1700 \mathrm{~h}$. Administration of the supplement was at approximately 0600, 1400, and $2000 \mathrm{~h}$. Despite the relatively large volume of fluids [ 4 quarts $(3.8 \mathrm{~L})$ of milk replacer and 6 quarts $(5.7 \mathrm{~L})$ of supplement] being offered over only a $14-\mathrm{h}$ interval, refusal or loss of appetite never necessitated administration of milk or supplement via esophageal feeder. Only 4 calves consumed as little as $50 \%$ of daily milk feeding (esophageal feeding was to occur if $<50 \%$ ). These 4 calves were equally distributed between treatment groups. This would seem to demonstrate that dehydrated calves will voluntarily consume a relatively large volume of fluids, and concerns of refusal or need to administer via esophageal feeder are unjustified. If a calf does not readily consume milk or the supplement, the calf should be assessed to see if it is either not dehydrated or has lost a suckle reflex due to severity of dehydration and acidemia.

Prevention, management, and treatment of diarrhea in preweaned calves is of great importance to animal health and the economics of an operation. Best management incorporates a multimodal, preventive approach that focuses on decreasing disease burden and supporting calf immunity, as well as implementing treatment protocols that allow for early detection and early action. Protocols should be designed to allow rapid, consistent rehydration, and be user-friendly and efficient. This study indicates that calves that were moderately dehydrated by an induced hyperosmotic diarrhea were effectively rehydrated by both i.v. LRS as well as a commercial nutritional supplement. Having a proven, effective oral rehydrating supplement as an effective treatment option gives producers a safe and easy to use alternative to administering i.v. fluids. This protocol can enable them to better treat diarrhea and prevent calfhood mortality.

\section{ACKNOWLEDGMENTS}

Supported in part by a grant from Boehringer-Ingelheim Vetmedica Inc. (St. Joseph, MO). Additional support was provided by the Department of Veterinary Pathobiology, Oklahoma State University Center for Veterinary Health Sciences (Stillwater).

\section{REFERENCES}

Berchtold, J. 2009. Treatment of calf diarrhea: Intravenous fluid therapy. Vet. Clin. North Am. Food Anim. Pract. 25:73-99.

Constable, P. 2003. Fluid and electrolyte therapy in ruminants. Vet. Clin. North Am. Food Anim. Pract. 19:557-597. 
Constable, P. D. 2009. Treatment of calf diarrhea: Antimicrobial and ancillary treatments. Vet. Clin. North Am. Food Anim. Pract. 25:101-120.

Donovan, G. A., I. R. Dohoo, D. M. Montgomery, and F. L. Bennett. 1998. Calf and disease factors affecting growth in female Holstein calves in Florida, USA. Prev. Vet. Med. 33:1-10.

Foster, D. M., and G. W. Smith. 2009. Pathophysiology of diarrhea in calves. Vet. Clin. North Am. Food Anim. Pract. 25:13-36.

Kasari, T. R. 1999. Metabolic acidosis in calves. Vet. Clin. North Am. Food Anim. Pract. 15:473-486.

Kasari, T. R., and J. M. Naylor. 1986. Further studies on the clinical features and clinicopathological findings of a syndrome of metabolic acidosis with minimal dehydration in neonatal calves. Can. J. Vet. Res. 50:502-508.

Leal, M. L., S. S. Fialho, F. C. Cyrillo, H. G. Bertagnon, E. L. Ortolani, and F. J. Benesi. 2012. Intravenous hypertonic saline solution $(7.5 \%)$ and oral electrolytes to treat of calves with noninfectious diarrhea and metabolic acidosis. J. Vet. Intern. Med. 26:1042-1050.

McGuirk, S. M. 2008. Disease management of dairy calves and heifers. Vet. Clin. North Am. Food Anim. Pract. 24:139-153.

Michell, A. R., H. W. Brooks, D. G. White, and A. J. Wagstaff. 1992. The comparative effectiveness of 3 commercial oral solutions in correcting fluid, electrolyte and acid-base disturbances caused by calf diarrhea. Br. Vet. J. 148:507-522.

Nakagawa, M., K. Suzuki, and K. Taguchi. 2007. Relationship between depression score and acid-base status in Japanese Black calves with diarrhea. J. Vet. Med. Sci. 69:549-552.
Naylor, J. M. 1999. Oral electrolyte therapy. Vet. Clin. North Am. Food Anim. Pract. 15:487-504.

Noritomi, D. T., A. J. Pereira, D. D. Bugano, P. S. Rehder, and E. Silva. 2011. Impact of Plasma-Lyte pH 7.4 on acid-base status and hemodynamics in a model of controlled hemorrhagic shock. Clinics (Sao Paulo, Brazil) 66:1969-1974.

Smith, G. 2015. Antimicrobial decision making for enteric diseases of cattle. Vet. Clin. North Am. Food Anim. Pract. 31:47-60.

Smith, G. W. 2009. Treatment of calf diarrhea: Oral fluid therapy. Vet. Clin. North Am. Food Anim. Pract. 25:55-72.

Smith, G. W., and J. Berchtold. 2014. Fluid therapy in calves. Vet. Clin. North Am. Food Anim. Pract. 30:409-427.

USDA. 2007. Dairy 2007, Part I: Reference of dairy cattle health and management practices in the United States. C. USDA-APHIS-VS. National Animal Health Monitoring System, Fort Collins, CO. N480.1007.

USDA. 2010. Beef 2007-08, Part IV: Reference of beef cow-calf management practices in the United States. C. USDA-APHIS-VS. Fort Collins, CO. \#523.0210.

Walker, P. G., P. D. Constable, D. E. Morin, J. K. Drackley, J. H Foreman, and J. C. Thurmon. 1998. A reliable, practical, and economical protocol for inducing diarrhea and severe dehydration in the neonatal calf. Can. J. Vet. Res. 62:205-213. 